\title{
Open Journal of Mathematical
} Optimization

Salihah Alwadani, Heinz H. Bauschke, Julian P. Revalski \& Xianfu Wang

The difference vectors for convex sets and a resolution of the geometry conjecture

Volume 2 (2021), article no. 5 (18 pages)

https://doi.org/10.5802/ojmo.7

Article submitted on December 9, 2020, revised on May 27, 2021,

accepted on May 28, 2021.

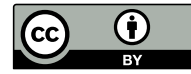

This article is licensed under the

Creative Commons AtTribution 4.0 International LiCEnSE.

http://creativecommons.org/licenses/by/4.0/ 


\title{
The difference vectors for convex sets and a resolution of the geometry conjecture
}

\author{
Salihah Alwadani \\ Mathematics \\ University of British Columbia \\ Kelowna, B.C. V1V 1V7 \\ Canada \\ saliha01@mail.ubc.ca \\ Heinz H. Bauschke \\ Mathematics \\ University of British Columbia \\ Kelowna, B.C. V1V 1V7 \\ Canada \\ heinz. bauschke@ubc.ca \\ Julian P. Revalski \\ Institute of Mathematics and Informatics \\ Bulgarian Academy of Sciences \\ Acad. G. Bonchev str., Block 8 \\ 1113 Sofia \\ Bulgaria \\ revalski@math.bas.bg \\ Xianfu Wang \\ Mathematics \\ University of British Columbia \\ Kelowna, B.C. V1V 1V7 \\ Canada \\ shawn.wang@ubc.ca
}

\begin{abstract}
The geometry conjecture, which was posed nearly a quarter of a century ago, states that the fixed point set of the composition of projectors onto nonempty closed convex sets in Hilbert space is actually equal to the intersection of certain translations of the underlying sets.

In this paper, we provide a complete resolution of the geometry conjecture. Our proof relies on monotone operator theory. We revisit previously known results and provide various illustrative examples. Comments on the numerical computation of the quantities involved are also presented.
\end{abstract}

Digital Object Identifier 10.5802/ojmo.7

Keywords Attouch-Théra duality, circular right shift operator, convex sets, cycle, fixed point set, monotone operator theory, projectors.

Acknowledgments The authors thank two referees and the editor for careful reading, thoughtful comments, and suggestions which significantly improved the presentation of the results. HHB and XW are supported by the Natural Sciences and Engineering Research Council of Canada.

\section{Introduction}

\subsection{Fixed points of compositions of projectors}

Throughout,

$X$ is a real Hilbert space

and

$C_{1}, \ldots, C_{m}$ are nonempty closed convex subsets of $X$

(i)
(c) Salihah Alwadani \& Heinz H. Bauschke \& Julian P. Revalski \& Xianfu Wang;
licensed under Creative Commons License Attribution 4.0 International

OJMO Volume 2 (2021), article no. 5 
with projectors $P_{C_{1}}, \ldots, P_{C_{m}}$ which we also write more simply as $P_{1}, \ldots, P_{m}$, and with $m \in\{2,3, \ldots\}$. We define the fixed point sets of the cyclic compositions by

$$
F_{m}:=\operatorname{Fix}\left(P_{m} \cdots P_{1}\right), \quad F_{m-1}:=\operatorname{Fix}\left(P_{m-1} \cdots P_{1} P_{m}\right), \quad \ldots \quad, F_{1}:=\operatorname{Fix}\left(P_{1} P_{m} \cdots P_{2}\right) .
$$

Compositions of projectors are often employed in projection methods. This is a vast area which we will not summarize here; however, we refer the reader to [16] as a starting point as well as the very recent paper [18].

\subsection{The geometry conjecture, difference vectors, and cycles}

The geometry conjecture, formulated first in 1997 (see [8, Conjecture 5.1.6]), states that there exists a list of vectors $v_{1}, \ldots, v_{m}$ in $X$ such that

$$
v_{1}+v_{2}+\cdots+v_{m}=0
$$

and

$$
F_{m}=C_{m} \cap\left(C_{m-1}+v_{m-1}\right) \cap \cdots \cap\left(C_{1}+v_{1}+\cdots+v_{m-1}\right),
$$

and analogously for $F_{m-1}, \ldots, F_{1}$. These vectors form the tuple $\left(v_{1}, \ldots, v_{m}\right)$ of difference vectors and they are sometimes also referred to as displacement vectors or gap vectors.

This conjecture is known to be true when $m=2$ or $C_{1} \cap C_{2} \cap \cdots \cap C_{m} \neq \varnothing$; see [8, Subsection 5.1]. The following is known when all sets $F_{i}$ are nonempty: let $f_{1} \in F_{1}$, and set $f_{2}:=P_{2} f_{1}, f_{3}:=P_{3} f_{2}, \ldots, f_{m}:=P_{m} f_{m-1}$; we shall refer to the tuple $\left(f_{1}, \ldots, f_{m}\right)$ as a cycle. (Cycles are of interest even when $C_{1}, \ldots, C_{m}$ are all hyperplanes, see [14, Chapter 8] and [15, Chapter 50].) Setting

$$
v_{1}=f_{2}-f_{1}, v_{2}=f_{3}-f_{2}, \ldots, v_{m-1}=f_{m}-f_{m-1}, v_{m}=f_{1}-f_{m},
$$

which turns out to be independent of the cycle chosen, makes (4) true and yields "one half" of (5), namely: $F_{m} \subseteq C_{m} \cap\left(C_{m-1}+v_{m-1}\right) \cap \cdots \cap\left(C_{1}+v_{1}+\cdots+v_{m-1}\right)$ and analogously for $F_{m-1}, \ldots, F_{1}$ (see [8, Theorem 5.1.2]). However, this description is not fully satisfying - it is only implicit in the sense it was not known what the difference vectors are when the fixed point sets $F_{i}$ are empty. The sole exception to this mystery was the case when $m=2$ which allowed for the explicit description of the two difference vectors by

$$
P_{\overline{C_{2}-C_{1}}}(0), \quad P_{\overline{C_{1}-C_{2}}}(0) \text {; }
$$

see [7, Lemmas 2.1 and 2.3]. Note that this description is not based on the fixed point sets $F_{1}, F_{2}$. In this particular case, these fixed point sets have the beautiful description (see [17, Theorem 2])

$$
F_{1}=\left\{x \in C_{1} \mid d_{C_{2}}(x)=\inf \left\|C_{1}-C_{2}\right\|\right\}, \quad F_{2}=\left\{x \in C_{2} \mid d_{C_{1}}(x)=\inf \left\|C_{1}-C_{2}\right\|\right\} ;
$$

moreover, the cycles $\left(f_{1}, f_{2}\right)$ are precisely the minimizers of the bivariate function

$$
X \times X \rightarrow \mathbb{R}:\left(x_{1}, x_{2}\right) \mapsto\left\|x_{1}-x_{2}\right\|+\iota_{C_{1}}\left(x_{1}\right)+\iota_{C_{2}}\left(x_{2}\right),
$$

where $d_{S}$ and $\iota_{S}$ denote the distance and indicator function of a subset $S$ of $X$, respectively. (See $[6,7,8,17]$ for much more on the case when $m=2$.) A referee also pointed out that when $m=2$ and $F_{1}=F_{2}=\varnothing$ one cannot expect uniqueness of the difference vectors as one may simply separate the sets even further.

The case when $m \geq 3$ is very interesting: The negative result of Baillon, Combettes, and Cominetti (see [4, Theorem 2.3]) states that when $X$ is at least two-dimensional, then there is no function $\varphi$ such that the cycles are precisely the minimizers of the function $\varphi\left(x_{1}, \ldots, x_{m}\right)+\iota_{C_{1}}\left(x_{1}\right)+\cdots+\iota_{C_{m}}\left(x_{m}\right)$. (When $m=2$, we can pick $\varphi\left(x_{1}, x_{2}\right)=\left\|x_{1}-x_{2}\right\|$ or even the differentiable function $\varphi\left(x_{1}, x_{2}\right)=\frac{1}{2}\left\|x_{1}-x_{2}\right\|^{2}$. For results on underrelaxed projectors, see also $[5,19]$.) Even when cycles exist, the "meaning" of the distance vector was not understood.

\subsection{Aim and outline of this paper}

The aim of this paper is to settle the geometry conjecture in the affirmative. The resolution depends on key results from monotone operator theory and yields a formula for the difference vectors. 
The remainder of this paper is organized as follows. In Section 2, we reformulate cycles and difference vectors in a product space using Attouch-Théra duality. The proof of the geometry conjecture is then presented in Section 3 (see Theorem 9). The cases $m=2, m=3$ are investigated in Section 4 and 5. Numerical considerations are presented in Section 6 and 7. The paper concludes with a summary and perspectives for future work in Section 8.

Notation is largely from [10] to which we also refer for background material on projections, convex analysis, and monotone operator theory. For valuable references on monotone operator theory see, e.g., [12, 13, 23, 24].

\section{The displacement of the circular right shift operator}

\subsection{Product space and Attouch-Théra duality}

From now on, we will also work in the product space

$$
\mathbf{X}:=X^{m}
$$

in which we set

$$
\mathbf{C}:=C_{1} \times \cdots \times C_{m} \quad \text { and } \quad \boldsymbol{\Delta}:=\{(x, \ldots, x) \in \mathbf{X} \mid x \in X\} .
$$

It is well known that the projectors onto these sets are given by

$$
P_{\mathbf{C}}\left(x_{1}, \ldots, x_{m}\right)=\left(P_{1} x_{1}, \ldots, P_{m} x_{m}\right)
$$

and

$$
P_{\boldsymbol{\Delta}}\left(x_{1}, \ldots, x_{m}\right)=\frac{1}{m}\left(\sum_{i=1}^{m} x_{i}, \ldots, \sum_{i=1}^{m} x_{i}\right)
$$

respectively (see, e.g., [10, Proposition 29.4 and Proposition 26.4(iii)]). Next, we define the circular right-shift operator

$$
\mathbf{R}: \mathbf{X} \rightarrow \mathbf{X}:\left(x_{1}, x_{2}, \ldots, x_{m}\right) \mapsto\left(x_{m}, x_{1}, x_{2}, \ldots, x_{m-1}\right) .
$$

Recall (see Section 1.2) that $\mathbf{z}=\left(z_{1}, \ldots, z_{m}\right) \in \mathbf{X}$ is a cycle if $z_{1}=P_{1} z_{m}, z_{2}=P_{2} z_{1}, \ldots, z_{m}=P_{m} z_{m-1}$, which can be elegantly reformulated in $\mathbf{X}$ as the fixed point equation

$$
\mathbf{z}=P_{\mathbf{C}}(\mathbf{R z}) .
$$

Denote the (possibly empty) set of all cycles by

$$
\mathbf{Z}:=\operatorname{Fix}\left(P_{\mathbf{C}} \mathbf{R}\right) .
$$

In passing, we note that if $Q_{i}:\left(x_{1}, \ldots, x_{m}\right) \rightarrow x_{i}$, then $F_{i}=Q_{i}(\mathbf{Z})$. Because $P_{\mathbf{C}}=\left(\operatorname{Id}+N_{\mathbf{C}}\right)^{-1}$, where $N_{\mathbf{C}}$ denotes the normal cone operator of $\mathbf{C}$, it follows that (15) is equivalent to $\mathbf{R z} \in\left(\operatorname{Id}+N_{\mathbf{C}}\right)(\mathbf{z})$ and to

$$
0 \in N_{\mathbf{C}}(\mathbf{z})+(\operatorname{Id}-\mathbf{R})(\mathbf{z}) .
$$

We view this last inclusion sum problem as primal (Attouch-Théra) problem for the pair $\left(N_{\mathbf{C}}\right.$,Id $-\mathbf{R}$ ). (See [3] and [9] for more on Attouch-Théra duality.) In view of the linearity of $\mathbf{R}$, the Attouch-Théra dual problem simplifies to

$$
0 \in N_{\mathbf{C}}^{-1}(\mathbf{y})+(\operatorname{Id}-\mathbf{R})^{-1}(\mathbf{y}) .
$$

If $\mathbf{z}$ is any cycle; equivalently, a solution to the primal problem (17), then a direct computation (or [9, Proposition 2.4 (iii)]) shows that $N_{\mathbf{C}}(\mathbf{z}) \cap-(\mathrm{Id}-\mathbf{R})(\mathbf{z})$ is a nonempty subset of dual solutions. Even better, both $N_{\mathbf{C}}$ and Id $-\mathbf{R}$ are paramonotone in the sense of Iusem [21] by, e.g., [10, Example 22.4(i) and Example 22.9]. It thus follows from [9, Theorem 5.3] that

$(\forall \mathbf{z} \in \mathbf{Z}) \quad \mathbf{R} \mathbf{z}-\mathbf{z}$ is the unique solution of (18)

and that

$$
\text { if } \mathbf{y} \text { solves (18), then } \mathbf{Z}=N_{\mathbf{C}}^{-1}(\mathbf{y}) \cap-(\operatorname{Id}-\mathbf{R})^{-1}(\mathbf{y}) \neq \varnothing \text {. }
$$




\section{$2.2(\mathrm{Id}-\mathbf{R})^{-1}$ and the skew operator $\mathbf{T}$}

Recall the definition of the circular right shift operator R (see (14)). By [1, Proposition 2.4], we have

$$
P_{\boldsymbol{\Delta}}=\frac{1}{m} \sum_{k=0}^{m-1} \mathbf{R}^{k}
$$

Now define

$$
\mathbf{T}=\frac{1}{2 m} \sum_{k=1}^{m-1}(m-2 k) \mathbf{R}^{k},
$$

which is a skew (hence maximally monotone) linear operator on $\mathbf{X}$, i.e.,

$$
\mathbf{T}^{*}=-\mathbf{T}
$$

with

$$
\operatorname{ran} \mathbf{T} \subseteq \boldsymbol{\Delta}^{\perp}
$$

(see [1, Proposition $3.2($ ii)\&(iii)]). Then [1, Theorem 3.3] states that

$$
(\mathrm{Id}-\mathbf{R})^{-1}=\frac{1}{2} \operatorname{Id}+N_{\boldsymbol{\Delta}^{\perp}}+\mathbf{T} .
$$

This form of $(\mathrm{Id}-\mathbf{R})^{-1}$ makes it clear that this operator is strongly monotone with constant $\frac{1}{2}$ which implies that

the dual problem (18) has at most one solution

which is consistent with (19). (A feature of Attouch-Théra duality is that either both primal and dual have solutions or they both don't. It is possible that there is no cycle and hence no dual solution; see Section 5.2.2.)

We now collect some useful identities.

- Proposition 1. We have $P_{\Delta} \mathbf{R}=\mathbf{R} P_{\boldsymbol{\Delta}}=P_{\boldsymbol{\Delta}}$ and hence $P_{\boldsymbol{\Delta}^{\perp}} \mathbf{R}=\mathbf{R}-P_{\boldsymbol{\Delta}}$.

Proof. Recalling (21), we observe that $P_{\Delta} \mathbf{R}=\mathbf{R} P_{\boldsymbol{\Delta}}$. Furthermore,

$$
\mathbf{R} P_{\boldsymbol{\Delta}}=\frac{1}{m} \sum_{k=0}^{m-1} \mathbf{R}^{k+1}=\frac{1}{m} \sum_{k=1}^{m} \mathbf{R}^{k}=\frac{1}{m} \sum_{k=0}^{m-1} \mathbf{R}^{k}=P_{\boldsymbol{\Delta}}
$$

because $\mathbf{R}^{m}=\mathbf{R}^{0}=$ Id. It follows that $P_{\boldsymbol{\Delta}^{\perp}} \mathbf{R}=\left(\operatorname{Id}-P_{\boldsymbol{\Delta}}\right) \mathbf{R}=\mathbf{R}-P_{\boldsymbol{\Delta}}$.

For the remainder of this section, let us abbreviate

$$
\mathbf{Q}:=\frac{1}{m} \sum_{k=1}^{m-1} k \mathbf{R}^{k}
$$

Clearly, $\mathbf{Q}$ commutes with $\mathbf{R}$, and hence also with $P_{\boldsymbol{\Delta}}$ by (21).

- Proposition 2. $2 \mathbf{Q} P_{\boldsymbol{\Delta}}=(m-1) P_{\boldsymbol{\Delta}}$.

Proof. Using Proposition 1, we see that

$$
\mathbf{Q} P_{\boldsymbol{\Delta}}=\frac{1}{m} \sum_{k=1}^{m-1} k \mathbf{R}^{k} P_{\boldsymbol{\Delta}}=\frac{1}{m} \sum_{k=1}^{m-1} k P_{\boldsymbol{\Delta}}=\frac{1}{m} \frac{(m-1) m}{2} P_{\boldsymbol{\Delta}}=\frac{m-1}{2} P_{\boldsymbol{\Delta}}
$$

as claimed.

Proposition 3. $-\mathbf{Q}(\operatorname{Id}-\mathbf{R})=P_{\Delta^{\perp}}$. 
Proof. Using (28) and (21), we obtain

$$
\begin{aligned}
-m \mathbf{Q}(\mathrm{Id}-\mathbf{R}) & =(\mathbf{R}-\mathrm{Id})(m \mathbf{Q})=(\mathbf{R}-\mathrm{Id}) \sum_{k=1}^{m-1} k \mathbf{R}^{k} \\
& =\sum_{k=1}^{m-1} k \mathbf{R}^{k+1}-\sum_{k=1}^{m-1} k \mathbf{R}^{k}=\sum_{k=2}^{m}(k-1) \mathbf{R}^{k}-\sum_{k=1}^{m-1} k \mathbf{R}^{k} \\
& =(m-1) \mathbf{R}^{m}+\left(\sum_{k=2}^{m-1}((k-1)-k) \mathbf{R}^{k}\right)-\mathbf{R} \\
& =(m-1) \operatorname{Id}-\left(\sum_{k=2}^{m-1} \mathbf{R}^{k}\right)-\mathbf{R} \\
& =m \operatorname{Id}-\sum_{k=0}^{m-1} \mathbf{R}^{k}=m \mathrm{Id}-m P_{\boldsymbol{\Delta}}=m P_{\boldsymbol{\Delta}^{\perp}}
\end{aligned}
$$

which completes the proof.

We are now ready for the main result of this section which will play a key role in subsequent sections.

- Theorem 4. We have

$$
\frac{1}{2} \mathrm{Id}+\mathbf{T}=\frac{m}{2} P_{\boldsymbol{\Delta}}-\mathbf{Q}
$$

and

$$
\left(\frac{1}{2} \operatorname{Id}+\mathbf{T}\right)^{-1}=\mathrm{Id}-\mathbf{R}+2 P_{\boldsymbol{\Delta}} .
$$

Proof. Using (22), (21), and (28), we have

$$
\mathbf{T}=\frac{1}{2 m} \sum_{k=1}^{m-1}(m-2 k) \mathbf{R}^{k}=\frac{1}{2} \sum_{k=1}^{m-1} \mathbf{R}^{k}-\frac{1}{m} \sum_{k=1}^{m-1} k \mathbf{R}^{k}=\frac{1}{2}\left(-\mathrm{Id}+m P_{\boldsymbol{\Delta}}\right)-\mathbf{Q}
$$

which gives (31).

Next, using (31), Proposition 1, Proposition 2, and Proposition 3, we obtain

$$
\begin{aligned}
\left(\frac{1}{2} \operatorname{Id}+\mathbf{T}\right)\left(\operatorname{Id}-\mathbf{R}+2 P_{\boldsymbol{\Delta}}\right) & =\left(\frac{m}{2} P_{\boldsymbol{\Delta}}-\mathbf{Q}\right)\left(\mathrm{Id}-\mathbf{R}+2 P_{\boldsymbol{\Delta}}\right) \\
& =\frac{m}{2}\left(P_{\boldsymbol{\Delta}}-P_{\boldsymbol{\Delta}} \mathbf{R}\right)+m P_{\boldsymbol{\Delta}}-\mathbf{Q}(\mathrm{Id}-\mathbf{R})-2 \mathbf{Q} P_{\boldsymbol{\Delta}} \\
& =m P_{\boldsymbol{\Delta}}+P_{\boldsymbol{\Delta}^{\perp}}-(m-1) P_{\boldsymbol{\Delta}}=P_{\boldsymbol{\Delta}}+P_{\boldsymbol{\Delta}^{\perp}} \\
& =\mathrm{Id} .
\end{aligned}
$$

This verifies (32) and thus completes the proof.

- Corollary 5. We have

$$
\left.\left(\frac{1}{2} \operatorname{Id}+T\right)^{-1}\right|_{\Delta^{\perp}}=\left.(\operatorname{Id}-\mathbf{R})\right|_{\Delta^{\perp}},
$$

Proof. From (32), we have $\left.\left(\frac{1}{2} \operatorname{Id}+T\right)^{-1}\right|_{\Delta^{\perp}}=\left.\left(\operatorname{Id}-\mathbf{R}+2 P_{\boldsymbol{\Delta}}\right)\right|_{\boldsymbol{\Delta}^{\perp}}=\left.(\operatorname{Id}-\mathbf{R})\right|_{\boldsymbol{\Delta}^{\perp}}$.

\section{The proof of the geometry conjecture}

Armed with (25), write the operator from the dual problem (18) as

$$
N_{\mathbf{C}}^{-1}+(\mathrm{Id}-\mathbf{R})^{-1}=N_{\mathbf{C}}^{-1}+\frac{1}{2} \operatorname{Id}+N_{\boldsymbol{\Delta}^{\perp}}+\mathbf{T} .
$$

This operator is in general not maximally monotone. On the other hand, $N_{\mathbf{C}}^{-1}+N_{\boldsymbol{\Delta}^{\perp}}=N_{\mathbf{C}}^{-1}+N_{\boldsymbol{\Delta}}^{-1}=$ $\partial \sigma_{\mathbf{C}}+\partial \sigma_{\boldsymbol{\Delta}} \subseteq \partial \sigma_{\mathbf{C}+\boldsymbol{\Delta}}$, where $\sigma_{\mathbf{S}}$ denotes the support function of a subset $\mathbf{S}$ of $\mathbf{X}$. Altogether, instead of working 
with (36), which has no solution if there are no cycles, we propose to work with the enlarged dual problem featuring the maximally and strongly monotone operator

$$
\frac{1}{2} \operatorname{Id}+\mathbf{T}+\partial \sigma_{\mathbf{C}+\boldsymbol{\Delta}} .
$$

Using, e.g., [10, Proposition 22.11(ii)], the corresponding inclusion problem always has a unique zero, which we denote by $\mathbf{y} \in \mathbf{X}$ :

$$
0 \in \frac{1}{2} \mathbf{y}+\mathbf{T} \mathbf{y}+\partial \sigma_{\mathbf{C}+\boldsymbol{\Delta}}(\mathbf{y}) .
$$

(In fact, $\mathbf{y}$ is the resolvent of the maximally monotone operator $2 \mathbf{T}+2 \partial \sigma_{\mathbf{C}+\boldsymbol{\Delta}}$, evaluated at 0 .) Note that, using [10, Proposition 6.49 and Example 11.2] $\mathbf{y} \in \operatorname{dom} \partial \sigma_{\mathbf{C}+\boldsymbol{\Delta}} \subseteq \operatorname{dom} \sigma_{\mathbf{C}+\boldsymbol{\Delta}}=\operatorname{dom}\left(\sigma_{\mathbf{C}}+\sigma_{\boldsymbol{\Delta}}\right)=\operatorname{dom} \sigma_{\mathbf{C}} \cap \operatorname{dom} \sigma_{\boldsymbol{\Delta}} \subseteq$ $(\operatorname{rec} \mathbf{C})^{\ominus} \cap(\operatorname{rec} \boldsymbol{\Delta})^{\ominus}$; thus,

$$
\mathbf{y} \in(\operatorname{rec} \mathbf{C})^{\ominus} \cap \boldsymbol{\Delta}^{\perp} .
$$

Now define

$$
\mathbf{e}:=-\frac{1}{2} \mathbf{y}-\mathbf{T y} \in \boldsymbol{\Delta}^{\perp},
$$

where $\mathbf{e} \in \boldsymbol{\Delta}^{\perp}$ because $\mathbf{y} \in \boldsymbol{\Delta}^{\perp}$ (see (39)) and $\operatorname{ran} \mathbf{T} \subseteq \boldsymbol{\Delta}^{\perp}$ (see (24)). Note that $-\mathbf{e}=\left(\frac{1}{2} \mathrm{Id}+\mathbf{T}\right) \mathbf{y}$. Hence (35) yields

$$
\mathbf{y}=(\operatorname{Id}-\mathbf{R})(-\mathbf{e})=\mathbf{R e}-\mathbf{e} .
$$

Note that (38) is equivalent to $\mathbf{e} \in \partial \sigma_{\mathbf{C}+\boldsymbol{\Delta}}(\mathbf{y})=\partial \iota_{\mathbf{C}+\boldsymbol{\Delta}}^{*}(\mathbf{y})$, and hence also to

$$
\mathbf{y} \in N_{\overline{\mathbf{C}+\Delta}}(\mathbf{e}),
$$

where the superscript "** denotes Fenchel conjugation. We pause here to record the following result which provides a certificate for $\mathbf{y}$ :

- Proposition 6 (a characterization of $\mathbf{y}$ ). The unique solution to (38) is the unique vector $\mathbf{y}$ satisfying the following:

$$
\mathbf{y} \in \boldsymbol{\Delta}^{\perp}, \quad-\frac{1}{2} \mathbf{y}-\mathbf{T y} \in \overline{\mathbf{C}+\boldsymbol{\Delta}}, \text { and }(\forall \mathbf{c} \in \mathbf{C})\langle\mathbf{c}, \mathbf{y}\rangle \leq-\frac{1}{2}\|\mathbf{y}\|^{2} .
$$

Proof. As seen, $\mathbf{y}$ solves (38) if and only if (42) holds with e defined in (40). The latter condition is equivalent to $\mathbf{e}=-\frac{1}{2} \mathbf{y}-\mathbf{T y} \in \overline{\mathbf{C}+\boldsymbol{\Delta}}$ and $(\forall(\mathbf{c}, \mathbf{d}) \in \mathbf{C} \times \boldsymbol{\Delta})\left\langle\mathbf{y}, \mathbf{c}+\mathbf{d}+\frac{1}{2} \mathbf{y}+\mathbf{T y}\right\rangle \leq 0$. Because $\mathbf{y} \in \boldsymbol{\Delta}^{\perp}$ (see (39)) and $\mathbf{T}$ is skew (see (23)), the last condition is indeed equivalent to (43).

Combining (40) and (42), we deduce that

$\mathbf{e} \in \Delta^{\perp} \cap \overline{\mathbf{C}+\Delta}$.

(This last intersection $\boldsymbol{\Delta}^{\perp} \cap \overline{\mathbf{C}+\boldsymbol{\Delta}}$ need not be a singleton as we can see by studying the case when $C_{1}=C_{2}=$ $\cdots=C_{m}=X$ and hence $\mathbf{C}=\mathbf{X}$, in which case the intersection is $\boldsymbol{\Delta}^{\perp}$.)

- Theorem 7. With $\mathbf{y}$ and $\mathbf{e}$ as defined in (38) and (40) respectively, the set of cycles is given by

$$
\begin{aligned}
\mathbf{Z} & =N_{\mathbf{C}}^{-1}(\mathbf{y}) \cap(\mathbf{e}+\boldsymbol{\Delta}) \\
& =\mathbf{e}+(\boldsymbol{\Delta} \cap(\mathbf{C}-\mathbf{e})) .
\end{aligned}
$$

Proof. First, $\mathbf{C} \subseteq \overline{\mathbf{C}+\boldsymbol{\Delta}}$ because $0 \in \boldsymbol{\Delta}$. Hence $(\forall \mathbf{c} \in \mathbf{C})\langle\mathbf{y}, \mathbf{c}-\mathbf{e}\rangle \leq 0$ by (42). It follows that

$$
\sigma_{\mathbf{C}}(\mathbf{y}) \leq\langle\mathbf{y}, \mathbf{e}\rangle=-\frac{1}{2}\|\mathbf{y}\|^{2},
$$

where the equality follows from (23) and (40).

Next, $\mathbf{y}$ might even solve the original dual (18) in which case $\mathbf{Z}$ is given by (20). Whether or not this is the case, we always have, using (25), (40), and (39),

$$
\begin{aligned}
N_{\mathbf{C}}^{-1}(\mathbf{y}) \cap-(\mathrm{Id}-\mathbf{R})^{-1}(\mathbf{y}) & =N_{\mathbf{C}}^{-1}(\mathbf{y}) \cap\left(-\frac{1}{2} \mathbf{y}-\mathbf{T} \mathbf{y}-N_{\boldsymbol{\Delta}^{\perp}}(\mathbf{y})\right) \\
& =N_{\mathbf{C}}^{-1}(\mathbf{y}) \cap(\mathbf{e}+\boldsymbol{\Delta}) .
\end{aligned}
$$


Altogether, this yields (45a).

Now let $\mathbf{x} \in \mathbf{X}$ and set $\mathbf{d}:=\mathbf{x}-\mathbf{e}$. Then, using (45a), (39), and (46), we have the equivalences

$$
\begin{aligned}
\mathbf{x} \in \mathbf{Z} & \Leftrightarrow \mathbf{x} \in N_{\mathbf{C}}^{-1}(\mathbf{y}) \cap(\mathbf{e}+\mathbf{\Delta}) \\
& \Leftrightarrow \mathbf{y} \in N_{\mathbf{C}}(\mathbf{x}) \text { and } \mathbf{x}-\mathbf{e} \in \mathbf{\Delta} \\
& \Leftrightarrow \mathbf{y} \in N_{\mathbf{C}}(\mathbf{d}+\mathbf{e}) \text { and } \mathbf{d} \in \mathbf{\Delta} \\
& \Leftrightarrow \mathbf{d} \in \boldsymbol{\Delta}, \mathbf{d}+\mathbf{e} \in \mathbf{C} \text {, and }(\forall \mathbf{c} \in \mathbf{C}) \quad\langle\mathbf{y}, \mathbf{c}-(\mathbf{d}+\mathbf{e})\rangle \leq 0 \\
& \Leftrightarrow \mathbf{d} \in \boldsymbol{\Delta}, \mathbf{d}+\mathbf{e} \in \mathbf{C} \text {, and }(\forall \mathbf{c} \in \mathbf{C}) \quad\langle\mathbf{y}, \mathbf{c}-\mathbf{e}\rangle \leq 0 \\
& \Leftrightarrow \mathbf{d} \in \boldsymbol{\Delta} \cap(\mathbf{C}-\mathbf{e}) \text { and } \sigma_{\mathbf{C}}(\mathbf{y}) \leq\langle\mathbf{y}, \mathbf{e}\rangle \\
& \Leftrightarrow \mathbf{x}-\mathbf{e} \in \boldsymbol{\Delta} \cap(\mathbf{C}-\mathbf{e}),
\end{aligned}
$$

and this gives $(45 \mathrm{~b})$.

- Corollary 8. The following hold:

1. (orthogonal decomposition of $\mathbf{Z}) P_{\boldsymbol{\Delta}^{\perp}}(\mathbf{Z}) \subseteq\{\mathbf{e}\}$ and $P_{\boldsymbol{\Delta}}(\mathbf{Z})=\boldsymbol{\Delta} \cap(\mathbf{C}-\mathbf{e})$.

2. $\mathbf{Z} \neq \varnothing \Leftrightarrow \mathbf{e} \in \mathbf{C}+\boldsymbol{\Delta}$.

3. If $\mathbf{e} \in \mathbf{C}+\boldsymbol{\Delta}$, say $\mathbf{e}=\mathbf{c}+\mathbf{d}$, where $\mathbf{c} \in \mathbf{C}$ and $\mathbf{d} \in \boldsymbol{\Delta}$, then $\mathbf{c} \in \mathbf{Z}$.

4. If $\mathbf{z} \in \mathbf{Z}$, then $\mathbf{e}=P_{\boldsymbol{\Delta}^{\perp} \mathbf{z}} \in(\mathbf{C}+\boldsymbol{\Delta}) \cap \boldsymbol{\Delta}^{\perp}$.

Proof. 1. Recall that $\mathbf{e} \in \boldsymbol{\Delta}^{\perp}$ by (40). Clearly, $\boldsymbol{\Delta} \cap(\mathbf{C}-\mathbf{e}) \subseteq \boldsymbol{\Delta}$. Using (45b), we obtain an orthogonal decomposition of $\mathbf{Z}$, with $\boldsymbol{\Delta}^{\perp}$ component $P_{\boldsymbol{\Delta}^{\perp}}(\mathbf{Z}) \subseteq\{\mathbf{e}\}$ and $P_{\boldsymbol{\Delta}}(\mathbf{Z})=\boldsymbol{\Delta} \cap(\mathbf{C}-\mathbf{e})$.

2. Indeed, using (45b), we have $\mathbf{Z} \neq \varnothing \Leftrightarrow \boldsymbol{\Delta} \cap(\mathbf{C}-\mathbf{e}) \neq \varnothing \Leftrightarrow(\exists \mathbf{c} \in \mathbf{C}) \mathbf{c}-\mathbf{e} \in \boldsymbol{\Delta} \Leftrightarrow \mathbf{e} \in \mathbf{C}+\boldsymbol{\Delta}$.

3. Indeed, $\mathbf{c}-\mathbf{e}=-\mathbf{d} \in(\boldsymbol{\Delta} \cap(\mathbf{C}-\mathbf{e}))$ and so $\mathbf{c} \in \mathbf{e}+(\boldsymbol{\Delta} \cap(\mathbf{C}-\mathbf{e}))=\mathbf{Z}$ using (45b).

4. Using 1 and (40), we obtain $\mathbf{e}=P_{\boldsymbol{\Delta}^{\perp} \mathbf{z}}=\mathbf{z}-P_{\boldsymbol{\Delta}} \mathbf{z} \in(\mathbf{Z}-\boldsymbol{\Delta}) \cap \boldsymbol{\Delta}^{\perp} \subseteq(\mathbf{C}+\boldsymbol{\Delta}) \cap \boldsymbol{\Delta}^{\perp}$.

At long last, we define

$$
\mathbf{v}:=\mathbf{R}^{*} \mathbf{e}-\mathbf{e} \in \Delta^{\perp}
$$

where $\mathbf{v} \in \boldsymbol{\Delta}^{\perp}$ because $\operatorname{ran}\left(\operatorname{Id}-\mathbf{R}^{*}\right)=\operatorname{ran}(\operatorname{Id}-\mathbf{R})=\boldsymbol{\Delta}^{\perp}$ by $[11$, Theorem $2.2(\mathrm{iv})]$. Also (41) yields

$$
\mathbf{v}=\mathbf{R}^{*} \mathbf{e}-\mathbf{e}=-\mathbf{R}^{*}(\mathbf{R e}-\mathbf{e})=-\mathbf{R}^{*} \mathbf{y},
$$

which in turn gives

$$
\mathbf{y}=-\mathbf{R v} \text {. }
$$

Because $\mathbf{R}^{*}$ is the circular left shift, (49) and (51) yield

$$
\begin{aligned}
\mathbf{v} & =\left(e_{2}-e_{1}, e_{3}-e_{2}, \ldots, e_{m}-e_{m-1}, e_{1}-e_{m}\right), \text { where } \mathbf{e}=\left(e_{1}, \ldots, e_{m}\right) \\
& =\left(-y_{2},-y_{3}, \ldots,-y_{m},-y_{1}\right), \text { where } \mathbf{y}=\left(y_{1}, \ldots, y_{m}\right) .
\end{aligned}
$$

We are now ready for our main result.

- Theorem 9 (The geometry conjecture is true). The vector $\mathbf{v}$ defined in (49) (see also (52)) is the sought-after difference vector (see Section 1.2).

Proof. We must verify (5).

First, let $z_{m} \in F_{m}$. Then $z_{m}$ is the $m$ th component of some cycle $\mathbf{z}$. Obviously, $\mathbf{z} \in \mathbf{C}$. By (45b), there exists $x \in X$ such that

$$
z_{1}=e_{1}+x, z_{2}=e_{2}+x, \ldots, z_{m-1}=e_{m-1}+x, z_{m}=e_{m}+x .
$$

Hence

$$
\begin{aligned}
z_{m} & \in C_{m} \\
z_{m} & =e_{m}+x=\left(e_{m-1}+x\right)+\left(e_{m}-e_{m-1}\right)=z_{m-1}+v_{m-1} \in C_{m-1}+v_{m-1} \\
z_{m} & =\left(e_{m-2}+x\right)+\left(e_{m-1}-e_{m-2}\right)+\left(e_{m}-e_{m-1}\right) \in C_{m-2}+v_{m-2}+v_{m-1} \\
& \vdots \\
z_{m} & \in C_{1}+v_{1}+v_{2}+\cdots+v_{m-1} .
\end{aligned}
$$


We deduce that

$$
F_{m} \subseteq C_{m} \cap\left(C_{m-1}+v_{m-1}\right) \cap \cdots \cap\left(C_{1}+v_{1}+\cdots+v_{m-1}\right) .
$$

We now tackle the converse inclusion. Let

$$
c_{m} \in C_{m} \cap\left(C_{m-1}+v_{m-1}\right) \cap \cdots \cap\left(C_{1}+v_{1}+\cdots+v_{m-1}\right) .
$$

So there exist $c_{1} \in C_{1}, \ldots, c_{m} \in C_{m}$ such that

$$
\begin{aligned}
c_{m} & =c_{m-1}+v_{m-1} \\
& =c_{m-2}+v_{m-2}+v_{m-1} \\
& \vdots \\
& =c_{2}+v_{2}+\cdots+v_{m-1} \\
& =c_{1}+v_{1}+v_{2}+\cdots+v_{m-1} .
\end{aligned}
$$

It follows that $c_{m-1}=c_{m-2}+v_{m-2}, \ldots, c_{2}=c_{1}+v_{1}$, and $c_{1}=c_{m}+v_{m}$ (because $c_{m}=c_{1}+v_{1}+v_{2}+\cdots v_{m-1}=$ $\left.c_{1}-v_{m}\right)$. Setting $\mathbf{c}:=\left(c_{1}, \ldots, c_{m}\right)$, we rewrite this as $\mathbf{c}=\mathbf{R} \mathbf{c}+\mathbf{R v}$. Using $(49), \mathbf{c}=\mathbf{R} \mathbf{c}+\mathbf{R}\left(\mathbf{R}^{*} \mathbf{e}-\mathbf{e}\right)=\mathbf{R} \mathbf{c}+\mathbf{e}-\mathbf{R e}$. Hence $(\operatorname{Id}-\mathbf{R})(\mathbf{c}-\mathbf{e})=0$ and thus $\mathbf{c}-\mathbf{e} \in \operatorname{ker}(\operatorname{Id}-\mathbf{R})=\boldsymbol{\Delta}$. It follows that $\mathbf{c}-\mathbf{e} \in \boldsymbol{\Delta} \cap(\mathbf{C}-\mathbf{e})$ and now (45b) yields

$$
\mathbf{c}=\mathbf{e}+(\mathbf{c}-\mathbf{e}) \in \mathbf{e}+(\boldsymbol{\Delta} \cap(\mathbf{C}-\mathbf{e}))=\mathbf{Z} .
$$

Therefore,

$$
c_{m} \in F_{m}
$$

which completes the proof of the geometry conjecture!

\section{The case when $m=2$}

Throughout this section, we assume that $m=2$.

\subsection{Revisiting known results}

It is instructive to revisit this case even if we know the answer already; moreover, we will discover a new formula for the difference vector $\mathbf{v}$. By (14) and (22), $\mathbf{R}^{*}=\mathbf{R}$ and $\mathbf{T}=0$. Hence (38) turns into $0 \in \frac{1}{2} \mathbf{y}+\partial \sigma_{\mathbf{C}+\boldsymbol{\Delta}}(\mathbf{y}) \Leftrightarrow$ $0 \in \frac{1}{2} \mathbf{y}+N_{\overline{\mathbf{C}+\boldsymbol{\Delta}}}^{-1}(\mathbf{y}) \Leftrightarrow-\frac{1}{2} \mathbf{y} \in N_{\overline{\mathbf{C}+\boldsymbol{\Delta}}}^{-1}(\mathbf{y}) \Leftrightarrow \mathbf{y} \in N_{\overline{\mathbf{C}+\boldsymbol{\Delta}}}\left(-\frac{1}{2} \mathbf{y}\right) \Leftrightarrow \frac{1}{2} \mathbf{y} \in\left(\mathrm{Id}+N_{\overline{\mathbf{C}+\boldsymbol{\Delta}}}\right)\left(-\frac{1}{2} \mathbf{y}\right) \Leftrightarrow-\frac{1}{2} \mathbf{y}=P_{\overline{\mathbf{C}+\boldsymbol{\Delta}}}\left(\frac{1}{2} \mathbf{y}\right)$ $\Leftrightarrow\left[-\frac{1}{2} \mathbf{y} \in \overline{\mathbf{C}+\boldsymbol{\Delta}}\right.$ and $\left.(\forall(\mathbf{c}, \mathbf{d}) \in \mathbf{C} \times \boldsymbol{\Delta})\left\langle\mathbf{c}+\mathbf{d}-\left(-\frac{1}{2} \mathbf{y}\right), \frac{1}{2} \mathbf{y}-\left(-\frac{1}{2} \mathbf{y}\right)\right\rangle \leq 0\right] \Leftrightarrow[\mathbf{y} \in 2 \overline{(\boldsymbol{\Delta}-\mathbf{C})}$ and $(\forall(\mathbf{c}, \mathbf{d}) \in$ $\mathbf{C} \times \boldsymbol{\Delta})\langle 2(\mathbf{d}-\mathbf{c})-\mathbf{y}), 0-\mathbf{y}\rangle \leq 0] \Leftrightarrow$

$$
\mathbf{y}=P_{2 \overline{(\boldsymbol{\Delta}-\mathbf{C})}}(0) \text {. }
$$

By (40),

$$
\mathbf{e}=-\frac{1}{2} \mathbf{y}-0 \mathbf{y}=-\frac{1}{2} P_{2 \overline{(\boldsymbol{\Delta}-\mathbf{C})}}(0)=P_{-\overline{(\boldsymbol{\Delta}-\mathbf{C})}}(0)=P_{\overline{\mathbf{C}+\boldsymbol{\Delta}}}(0) .
$$

Finally, by (49),

$$
\mathbf{v}=-\mathbf{R}^{*} \mathbf{y}=-\mathbf{R y} .
$$

We now express these quantities also in the underlying space $X$. We claim that

$$
\mathbf{y} \stackrel{?}{=}\left(P_{\overline{C_{2}-C_{1}}}(0), P_{\overline{C_{1}-C_{2}}}(0)\right)=\left(P_{\overline{C_{2}-C_{1}}}(0),-P_{\overline{C_{2}-C_{1}}}(0)\right) .
$$

Set $y:=P_{\overline{C_{2}-C_{1}}}(0)$. Then $y \leftarrow c_{2, n}-c_{1, n}$, where $\left(c_{1, n}, c_{2, n}\right)_{n \in \mathbb{N}}$ is a sequence in $C_{1} \times C_{2}$. Now for every $n \in \mathbb{N}$, $c_{2, n}-c_{1, n}=2\left(\frac{1}{2}\left(c_{1, n}+c_{2, n}\right)-c_{1, n}\right)$ and $c_{1, n}-c_{2, n}=2\left(\frac{1}{2}\left(c_{1, n}+c_{2, n}\right)-c_{2, n}\right)$, so

$$
\left(c_{2, n}-c_{1, n}, c_{1, n}-c_{2, n}\right) \in 2(\boldsymbol{\Delta}-\mathbf{C})
$$


which implies $(y,-y) \in 2 \overline{(\boldsymbol{\Delta}-\mathbf{C})}$. Next, let us take $(\mathbf{c}, \mathbf{d}) \in \mathbf{C} \times \boldsymbol{\Delta}$, say $\mathbf{c}=\left(c_{1}, c_{2}\right) \in C_{1} \times C_{2}$ and $\mathbf{d}=(x, x)$ for some $x \in X$. Then

$$
\begin{aligned}
\langle 2(\mathbf{d}-\mathbf{c})-(y,-y), \mathbf{0}-(y,-y)\rangle & =\left\langle 2\left(x-c_{1}, x-c_{2}\right)-(y,-y),(-y, y)\right\rangle \\
& =\left\langle\left(2 x-2 c_{1}-y, 2 x-2 c_{2}+y\right),(-y, y)\right\rangle \\
& =\left\langle 2 x-2 c_{1}-y,-y\right\rangle+\left\langle 2 x-2 c_{2}+y, y\right\rangle \\
& =\left\langle 2 c_{2}-2 c_{1}-2 y,-y\right\rangle \\
& =2\left\langle\left(c_{2}-c_{1}\right)-y, 0-y\right\rangle \\
& \leq 0
\end{aligned}
$$

by definition of $y$. We have verified

$$
\mathbf{y}=\left(P_{\overline{C_{2}-C_{1}}}(0), P_{\overline{C_{1}-C_{2}}}(0)\right) .
$$

It follows (by (61) and (66)) that

$$
\mathbf{e}=-\frac{1}{2} \mathbf{y}=-\frac{1}{2}\left(P_{\overline{C_{2}-C_{1}}}(0), P_{\overline{C_{1}-C_{2}}}(0)\right)=\frac{1}{2}\left(P_{\overline{C_{1}-C_{2}}}(0), P_{\overline{C_{2}-C_{1}}}(0)\right)
$$

and (by (62) and (66))

$$
\mathbf{v}=-\mathbf{R y}=-\left(P_{\overline{C_{1}-C_{2}}}(0), P_{\overline{C_{2}-C_{1}}}(0)\right)=\left(P_{\overline{C_{2}-C_{1}}}(0), P_{\overline{C_{1}-C_{2}}}(0)\right)=\mathbf{y} .
$$

Hence

$$
v_{1}=P_{\overline{C_{2}-C_{1}}}(0) \text { and } v_{2}=P_{\overline{C_{1}-C_{2}}}(0)=-v_{1}
$$

and this is completely consistent with the known theory exposed in Section 1.2 (see (7))! Along our journey, we have thus discovered a new identity for $\mathbf{v}$ by combining (60) with (68) which we record in the following result.

- Proposition 10. $\mathbf{v}=\left(P_{\overline{C_{2}-C_{1}}}(0), P_{\overline{C_{1}-C_{2}}}(0)\right)=P_{2 \overline{\Delta-\mathbf{C}}}(0)=2 P_{\overline{\mathbf{\Delta}-\mathbf{C}}}(0)$.

\subsection{Two lines}

It is instructive to consider two general lines in $X$, given by

$$
C_{1}=c_{1}+\mathbb{R} u_{1}, C_{2}=c_{2}+\mathbb{R} u_{2}, \quad \text { where } c_{1} \perp u_{1}, c_{2} \perp u_{2} \text {, and }\left\|u_{1}\right\|=\left\|u_{2}\right\|=1
$$

because we will obtain descriptions of $\mathbf{Z}, \mathbf{v}, \mathbf{y}$, and $\mathbf{e}$. We start by noting that for every $i \in\{1,2\}$,

$$
(\forall x \in X) P_{i}(x)=c_{i}+\left\langle u_{i}, x\right\rangle u_{i} .
$$

Let $\mathbf{z}=\left(z_{1}, z_{2}\right) \in C_{1} \times C_{2}$. Then $z_{1}=c_{1}+\rho_{1} u_{1}$ and $z_{2}=c_{2}+\rho_{2} u_{2}$ for some $\rho_{1}, \rho_{2}$ in $\mathbb{R}$. Now assume that $\mathbf{z}$ is actually a cycle. Then $z_{2}=P_{2} P_{1} z_{2}$, i.e.,

$$
\begin{aligned}
c_{2}+\rho_{2} u_{2} & =z_{2} \\
& =P_{2} P_{1} z_{2} \\
& =c_{2}+\left\langle u_{2}, P_{1} z_{2}\right\rangle u_{2} \\
& =c_{2}+\left\langle u_{2}, c_{1}+\left\langle u_{1}, z_{2}\right\rangle u_{1}\right\rangle u_{2} \\
& =c_{2}+\left(\left\langle u_{2}, c_{1}\right\rangle+\left\langle u_{1}, z_{2}\right\rangle\left\langle u_{2}, u_{1}\right\rangle\right) u_{2} \\
& =c_{2}+\left(\left\langle u_{2}, c_{1}\right\rangle+\left\langle u_{1}, c_{2}+\rho_{2} u_{2}\right\rangle\left\langle u_{2}, u_{1}\right\rangle\right) u_{2} \\
& =c_{2}+\left(\left\langle u_{2}, c_{1}\right\rangle+\left\langle u_{1}, c_{2}\right\rangle\left\langle u_{2}, u_{1}\right\rangle+\rho_{2}\left\langle u_{1}, u_{2}\right\rangle\left\langle u_{2}, u_{1}\right\rangle\right) u_{2} ;
\end{aligned}
$$

equivalently,

$$
\rho_{2}\left(1-\left\langle u_{1}, u_{2}\right\rangle^{2}\right)=\left\langle c_{1}, u_{2}\right\rangle+\left\langle u_{1}, u_{2}\right\rangle\left\langle u_{1}, c_{2}\right\rangle .
$$

The theory bifurcates from here as we will see in the following subsections. 


\subsubsection{The lines are parallel}

Let's first assume that the two lines $C_{1}, C_{2}$ are parallel; equivalently, $\left\langle u_{1}, u_{2}\right\rangle^{2}=1$. Without loss of generality, $u_{2}=u_{1}=: u$. Then every $\rho_{2}$ in $\mathbb{R}$ solves (73). It then follows that the set of cycles is

$$
\mathbf{Z}=\left(c_{1}, c_{2}\right)+\mathbb{R}(u, u) .
$$

Moreover, using (6), (68), and (67), we obtain

$$
\mathbf{v}=\left(c_{2}-c_{1}, c_{1}-c_{2}\right)=\mathbf{y} \text { and } \mathbf{e}=\frac{1}{2}\left(c_{1}-c_{2}, c_{2}-c_{1}\right) .
$$

\subsubsection{The lines are not parallel}

Now we assume that $C_{1}, C_{2}$ are not parallel. Then $\left\langle u_{1}, u_{2}\right\rangle^{2}<1$ and solving (73) for $\rho_{2}$ yields

$$
\rho_{2}:=\frac{\left\langle u_{2}, c_{1}\right\rangle+\left\langle u_{1}, u_{2}\right\rangle\left\langle u_{1}, c_{2}\right\rangle}{1-\left\langle u_{1}, u_{2}\right\rangle^{2}}
$$

and analogously

$$
\rho_{1}:=\frac{\left\langle u_{1}, c_{2}\right\rangle+\left\langle u_{1}, u_{2}\right\rangle\left\langle u_{2}, c_{1}\right\rangle}{1-\left\langle u_{1}, u_{2}\right\rangle^{2}} .
$$

Hence the set of cycles $\mathbf{Z}$ has only one element, namely

$$
\mathbf{z}=\left(z_{1}, z_{2}\right)=\left(c_{1}+\rho_{1} u_{1}, c_{2}+\rho_{2} u_{2}\right) ;
$$

and $\mathbf{v}=\left(z_{2}-z_{1}, z_{1}-z_{2}\right)=\mathbf{y}$ and $\mathbf{e}=-\frac{1}{2} \mathbf{v}$ which we don't expand as the expressions don't simplify.

\section{The case when $m=3$}

Throughout this section, we assume that $m=3$. Then the matrix representations for $\mathbf{T}$ (see (22)) is

$$
\mathbf{T}=\frac{1}{6}\left(\begin{array}{lll}
0 & 0 & 1 \\
1 & 0 & 0 \\
0 & 1 & 0
\end{array}\right)-\frac{1}{6}\left(\begin{array}{lll}
0 & 1 & 0 \\
0 & 0 & 1 \\
1 & 0 & 0
\end{array}\right)=\frac{1}{6}\left(\begin{array}{rrr}
0 & -1 & 1 \\
1 & 0 & -1 \\
-1 & 1 & 0
\end{array}\right)
$$

and thus

$$
-\frac{1}{2} \mathrm{Id}-\mathbf{T}=\frac{1}{6}\left(\begin{array}{rrr}
-3 & 1 & -1 \\
-1 & -3 & 1 \\
1 & -1 & -3
\end{array}\right) .
$$

Thanks to (80), Proposition 6, (40), and (52), we obtain the following result:

- Theorem 11. Let $\mathbf{y}=\left(y_{1}, y_{2}, y_{3}\right) \in \mathbf{X}=X^{3}$. Then $\mathbf{y}$ is the unique solution of (38) if and only if all of the following hold:

$$
y_{1}+y_{2}+y_{3}=0,
$$

there exist sequences $\left(c_{1, n}\right)_{n \in \mathbb{N}}$ in $C_{1},\left(c_{2, n}\right)_{n \in \mathbb{N}}$ in $C_{2},\left(c_{3, n}\right)_{n \in \mathbb{N}}$ in $C_{3}$, and $\left(x_{n}\right)_{n \in \mathbb{N}}$ in $X$ such that

$$
\begin{aligned}
& c_{1, n}+x_{n} \rightarrow \frac{1}{6}\left(-3 y_{1}+y_{2}-y_{3}\right), \\
& c_{2, n}+x_{n} \rightarrow \frac{1}{6}\left(-y_{1}-3 y_{2}+y_{3}\right), \\
& c_{3, n}+x_{n} \rightarrow \frac{1}{6}\left(y_{1}-y_{2}-3 y_{3}\right),
\end{aligned}
$$

and $\left(\forall\left(c_{1}, c_{2}, c_{3}\right) \in C_{1} \times C_{2} \times C_{3}\right)$

$$
\left\langle y_{1}, c_{1}\right\rangle+\left\langle y_{2}, c_{2}\right\rangle+\left\langle y_{3}, c_{3}\right\rangle \leq-\frac{1}{2}\left(\left\|y_{1}\right\|^{2}+\left\|y_{2}\right\|^{2}+\left\|y_{3}\right\|^{2}\right) .
$$

If $\mathbf{y}=\left(y_{1}, y_{2}, y_{3}\right)$ satisfies all these conditions, then

$$
\mathbf{e}=\left(e_{1}, e_{2}, e_{3}\right)=\frac{1}{6}\left(-3 y_{1}+y_{2}-y_{3},-y_{1}-3 y_{2}+y_{3}, y_{1}-y_{3}-3 y_{3}\right)
$$

and

$$
\mathbf{v}=-\left(y_{2}, y_{3}, y_{1}\right)
$$

are the vectors from (40) and (49), respectively. 
Note that if $\mathbf{v}=\left(v_{1}, v_{2}, v_{3}\right)$, then we can obtain $\mathbf{y}$ through (51):

$\mathbf{y}=-\mathbf{R v}=-\left(v_{3}, v_{1}, v_{2}\right)$.

Moreover, if desired, we can find e by combining (40) and (79).

\subsection{Three lines}

Let us consider three lines, which can be treated similar to two lines (see Section 4.2). (For brevity, we will omit full details on the somewhat tedious algebraic manipulations.) We assume that

$$
C_{1}=c_{1}+\mathbb{R} u_{1}, \quad C_{2}=c_{2}+\mathbb{R} u_{2}, \quad C_{3}=c_{3}+\mathbb{R} u_{3},
$$

where

$$
c_{1} \perp u_{1}, \quad c_{2} \perp u_{2}, \quad c_{3} \perp u_{3} \quad \text { and } \quad\left\|u_{1}\right\|=\left\|u_{2}\right\|=\left\|u_{3}\right\|=1 .
$$

\subsubsection{All three lines are parallel}

Let's first assume that all lines are parallel; equivalently, $\left\langle u_{3}, u_{2}\right\rangle\left\langle u_{2}, u_{1}\right\rangle\left\langle u_{1}, u_{3}\right\rangle=1$. Without loss of generality, $u:=u_{1}=u_{2}=u_{3}$. Then the set of cycles is

$$
\mathbf{Z}=\left(c_{1}, c_{2}, c_{3}\right)+\mathbb{R}(u, u, u)
$$

and thus the difference vector is

$$
\mathbf{v}=\left(c_{2}-c_{1}, c_{3}-c_{2}, c_{1}-c_{3}\right) .
$$

In Figure 1, we visualize this case for three lines in $\mathbb{R}^{3}$.

\subsubsection{Not all three lines are parallel}

The case when not all lines are parallel corresponds to $\left\langle u_{3}, u_{2}\right\rangle\left\langle u_{2}, u_{1}\right\rangle\left\langle u_{1}, u_{3}\right\rangle<1$. Then the set of cycles $\mathbf{Z}$ consists is a singleton containing

$$
\mathbf{z}=\left(z_{1}, z_{2}, z_{3}\right)=\left(c_{1}+\rho_{1} u_{1}, c_{2}+\rho_{2} u_{2}, c_{3}+\rho_{3} u_{3}\right),
$$

where

$$
\begin{aligned}
\rho_{1} & :=\frac{\left\langle u_{1}, c_{3}\right\rangle+\left\langle u_{1}, u_{3}\right\rangle\left\langle u_{3}, c_{2}\right\rangle+\left\langle u_{1}, u_{3}\right\rangle\left\langle u_{3}, u_{2}\right\rangle\left\langle u_{2}, c_{1}\right\rangle}{1-\left\langle u_{3}, u_{2}\right\rangle\left\langle u_{2}, u_{1}\right\rangle\left\langle u_{1}, u_{3}\right\rangle}, \\
\rho_{2} & :=\frac{\left\langle u_{2}, c_{1}\right\rangle+\left\langle u_{2}, u_{1}\right\rangle\left\langle u_{1}, c_{3}\right\rangle+\left\langle u_{2}, u_{1}\right\rangle\left\langle u_{1}, u_{3}\right\rangle\left\langle u_{3}, c_{2}\right\rangle}{1-\left\langle u_{3}, u_{2}\right\rangle\left\langle u_{2}, u_{1}\right\rangle\left\langle u_{1}, u_{3}\right\rangle}, \\
\rho_{3} & :=\frac{\left\langle u_{3}, c_{2}\right\rangle+\left\langle u_{3}, u_{2}\right\rangle\left\langle u_{2}, c_{1}\right\rangle+\left\langle u_{3}, u_{2}\right\rangle\left\langle u_{2}, u_{1}\right\rangle\left\langle u_{1}, c_{3}\right\rangle}{1-\left\langle u_{3}, u_{2}\right\rangle\left\langle u_{2}, u_{1}\right\rangle\left\langle u_{1}, u_{3}\right\rangle},
\end{aligned}
$$

and

$$
\mathbf{v}=\left(z_{2}-z_{1}, z_{3}-z_{2}, z_{1}-z_{3}\right) .
$$

In Figure 2, we visualize this case for three lines in $\mathbb{R}^{3}$.

\subsection{An example featuring the epigraph of the exponential function}

In this section, we specialize further to

$$
X=\mathbb{R}^{2} .
$$

Inspired by [20, Section 3], we will present three sets in the Euclidean plane and consider two different orderings. The sets are the epigraph of the exponential function,

$$
\operatorname{epi}(\exp )=\left\{(\xi, \eta) \in \mathbb{R}^{2} \mid \exp (\xi) \leq \eta\right\}=\operatorname{gra}(\exp )+(\{0\} \times \mathbb{R}),
$$




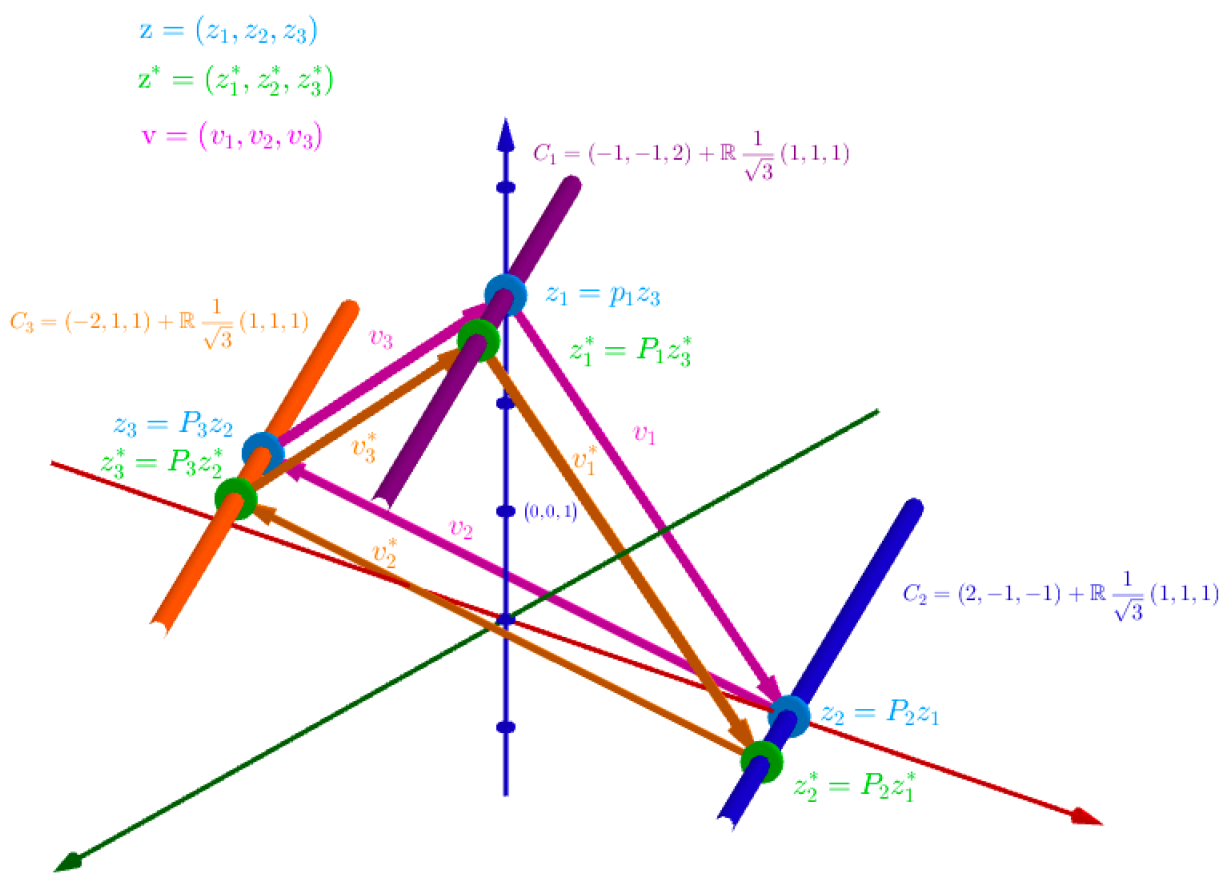

Figure 1 Visualization of the cycles and the difference vectors for three parallel lines in $\mathbb{R}^{3}$. See Section 5.1.1 for details.

along with the two horizontal lines

$\mathbb{R} \times\{0\}$ and $\mathbb{R} \times\{1\}$.

In the following we describe two orderings, one leading to the presence of cycles, the other to their absence. The case when there are cycles is depicted in Figure 3.

\subsubsection{An ordering with cycles}

In this section, we assume that

$$
C_{1}=\mathbb{R} \times\{0\}, \quad C_{2}=\mathbb{R} \times\{1\}, \quad C_{3}=\operatorname{epi}(\exp ) .
$$

Now set

$$
\mathbf{y}=\left(y_{1}, y_{2}, y_{3}\right)=((0,1),(0,-1),(0,0)) .
$$

We claim that (98) satisfies the characterization provided by Theorem 11.

Clearly, $y_{1}+y_{2}+y_{3}=(0+0+0,1-1+0)=(0,0)$ and so (81) holds.

Next, set $c_{1, n} \equiv(0,0) \in C_{1}, c_{2, n} \equiv(0,1) \in C_{2}, c_{3, n} \equiv(0,1)=(0, \exp (0)) \in C_{3}$, and $x_{n} \equiv\left(0,-\frac{2}{3}\right) \in X$. Then

$$
\begin{aligned}
& c_{1, n}+x_{n} \equiv\left(0,-\frac{2}{3}\right)=\frac{1}{6}(-3(0,1)+(0,-1)-(0,0))=\frac{1}{6}\left(-3 y_{1}+y_{2}-y_{3}\right), \\
& c_{2, n}+x_{n} \equiv\left(0, \frac{1}{3}\right)=\frac{1}{6}(-(0,1)-3(0,-1)+(0,0))=\frac{1}{6}\left(-y_{1}-3 y_{2}+y_{3}\right), \\
& c_{3, n}+x_{n} \equiv\left(0, \frac{1}{3}\right)=\frac{1}{6}((0,1)-(0,-1)-3(0,0))=\frac{1}{6}\left(y_{1}-y_{2}-3 y_{3}\right),
\end{aligned}
$$

and thus (82) holds. 


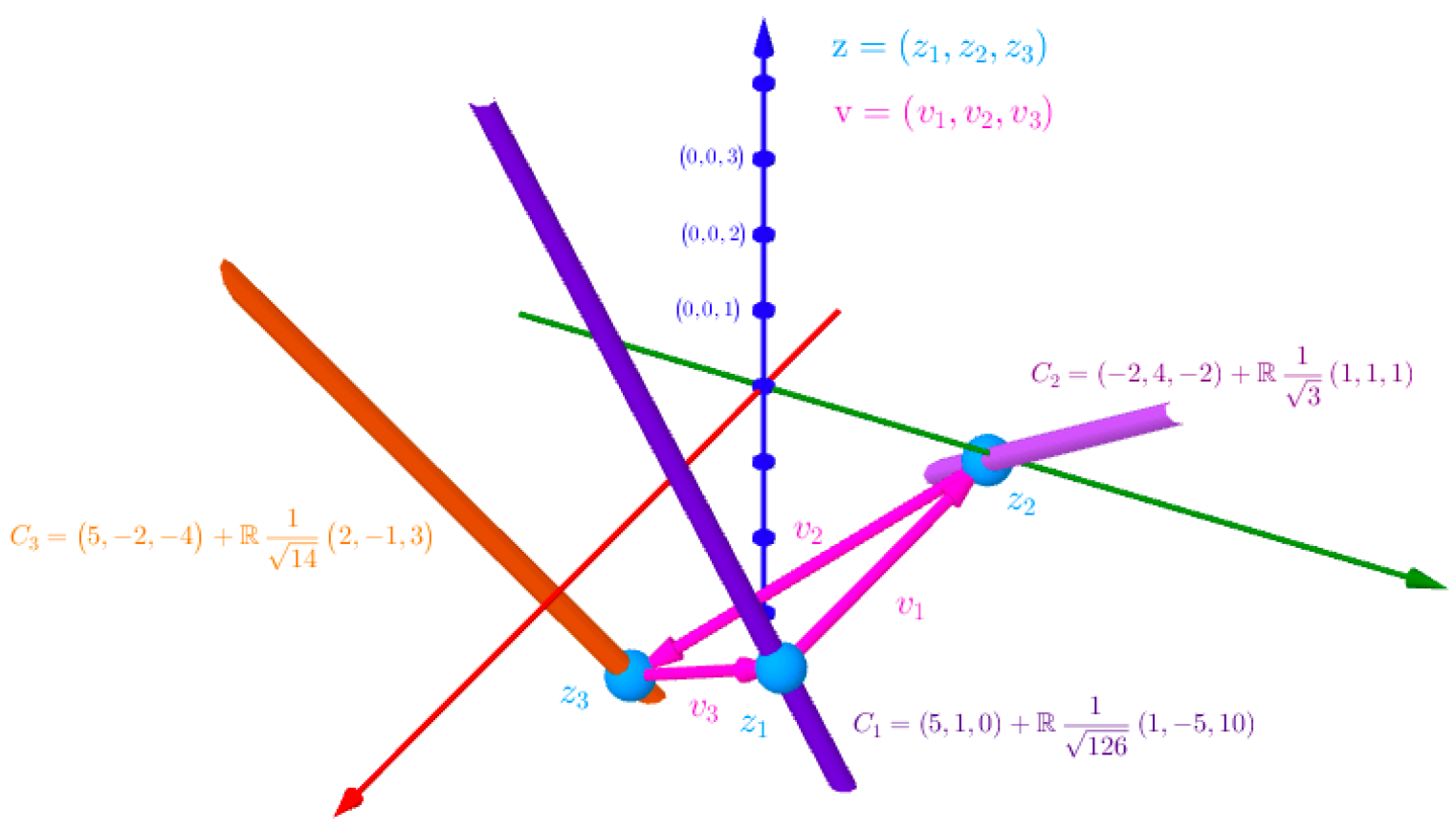

Figure 2 Visualization of the cycle and the difference vectors for three lines in $\mathbb{R}^{3}$ that are not parallel. See Section 5.1.2 for details.

Now let $c_{1}=\left(\gamma_{1}, 0\right) \in C_{1}, c_{2}=\left(\gamma_{2}, 1\right) \in C_{2}$, and $c_{3}=\left(\gamma_{3}, \exp \left(\gamma_{3}\right)+\delta_{3}\right) \in C_{3}$, where $\left\{\gamma_{1}, \gamma_{2}, \gamma_{3}\right\} \subseteq \mathbb{R}$, and $\delta_{3} \in \mathbb{R}_{+}$. Then

$$
\begin{aligned}
\left\langle y_{1}, c_{1}\right\rangle+\left\langle y_{2}, c_{2}\right\rangle+\left\langle y_{3}, c_{3}\right\rangle & =\left\langle(0,1),\left(\gamma_{1}, 0\right)\right\rangle+\left\langle(0,-1),\left(\gamma_{2}, 1\right)\right\rangle+\left\langle(0,0),\left(\gamma_{3}, \exp \left(\gamma_{3}\right)+\delta_{3}\right)\right\rangle \\
& =-1 \\
& =-\frac{1}{2}(1+1+0) \\
& =-\frac{1}{2}\left(\|(0,1)\|^{2}+\|(0,-1)\|^{2}+\|(0,0)\|^{2}\right) \\
& =-\frac{1}{2}\left(\left\|y_{1}\right\|^{2}+\left\|y_{2}\right\|^{2}+\left\|y_{3}\right\|^{2}\right) .
\end{aligned}
$$

and thus (83) holds (even with equality).

Next, using (84) and (85), we obtain

$$
\begin{aligned}
& \mathbf{e}=\left(e_{1}, e_{2}, e_{3}\right)=\left(\left(0,-\frac{2}{3}\right),\left(0, \frac{1}{3}\right),\left(0, \frac{1}{3}\right)\right) \\
& \mathbf{v}=\left(v_{1}, v_{2}, v_{3}\right)=((0,1),(0,0),(0,-1)) .
\end{aligned}
$$

The vector $\mathbf{v}$ allows us to find the fixed point sets $F_{1}, F_{2}, F_{3}$ (see (3)) via Theorem 9. For instance,

$$
\begin{aligned}
F_{3} & =C_{3} \cap\left(C_{2}+v_{2}\right) \cap\left(C_{1}+v_{1}+v_{2}\right) \\
& =\operatorname{epi}(\exp ) \cap(\mathbb{R} \times\{1\}+(0,0)) \cap(\mathbb{R} \times\{0\}+(0,1)) \\
& =\operatorname{epi}(\exp ) \cap(\mathbb{R} \times\{1\}) \cap(\mathbb{R} \times\{1\}) \\
& =\mathbb{R}_{-} \times\{1\},
\end{aligned}
$$

which can also be seen geometrically. 


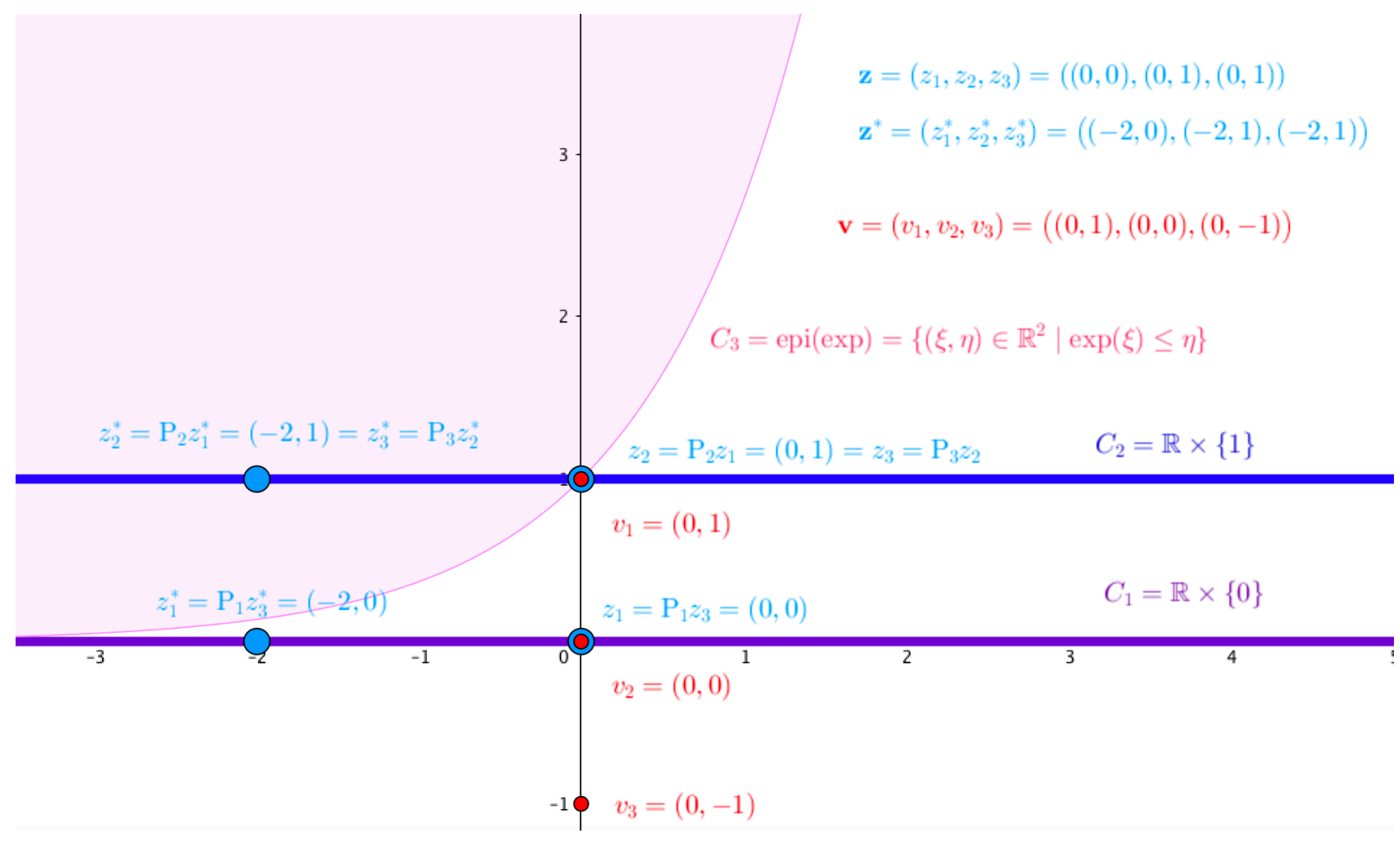

Figure 3 Visualization of the case of two lines and the epigraph of the exponential function where there are cycles. See Section 5.2 for details.

\subsubsection{An ordering without cycles}

In this section, we assume that

$$
C_{1}=\mathbb{R} \times\{1\}, \quad C_{2}=\mathbb{R} \times\{0\}, \quad C_{3}=\operatorname{epi}(\exp ),
$$

which is nearly the same set up as in the last, the crucial difference is that $C_{1}$ and $C_{2}$ were interchanged! Now set

$$
\mathbf{y}=\left(y_{1}, y_{2}, y_{3}\right)=((0,-1),(0,1),(0,0))
$$

We claim that (107) satisfies the characterization provided by Theorem 11.

Clearly, $y_{1}+y_{2}+y_{3}=(0+0+0,-1+1+0)=(0,0)$ and so $(81)$ holds.

Next, set $(\forall n \in \mathbb{N}) c_{1, n}=(-n, 1) \in C_{1}, c_{2, n}=(-n, 0) \in C_{2}, c_{3, n}=\left(-n, \exp (-n) \in C_{3}\right.$, and $x_{n}=\left(n,-\frac{1}{3}\right) \in$ $X$. Then

$$
\begin{aligned}
c_{1, n}+x_{n} & \equiv\left(0, \frac{2}{3}\right)=\frac{1}{6}(-3(0,-1)+(0,1)-(0,0))=\frac{1}{6}\left(-3 y_{1}+y_{2}-y_{3}\right), \\
c_{2, n}+x_{n} & \equiv\left(0,-\frac{1}{3}\right)=\frac{1}{6}(-(0,-1)-3(0,1)+(0,0))=\frac{1}{6}\left(-y_{1}-3 y_{2}+y_{3}\right), \\
c_{3, n}+x_{n} & =\left(0, \exp (-n)-\frac{1}{3}\right) \\
& \rightarrow\left(0,-\frac{1}{3}\right)=\frac{1}{6}((0,-1)-(0,1)-3(0,0))=\frac{1}{6}\left(y_{1}-y_{2}-3 y_{3}\right),
\end{aligned}
$$

and thus (82) holds. 
Now let $c_{1}=\left(\gamma_{1}, 1\right) \in C_{1}, c_{2}=\left(\gamma_{2}, 0\right) \in C_{2}$, and $c_{3}=\left(\gamma_{3}, \exp \left(\gamma_{3}\right)+\delta_{3}\right) \in C_{3}$, where $\left\{\gamma_{1}, \gamma_{2}, \gamma_{3}\right\} \subseteq \mathbb{R}$, and $\delta_{3} \in \mathbb{R}_{+}$. Then

$$
\begin{aligned}
\left\langle y_{1}, c_{1}\right\rangle+\left\langle y_{2}, c_{2}\right\rangle+\left\langle y_{3}, c_{3}\right\rangle & =\left\langle(0,-1),\left(\gamma_{1}, 1\right)\right\rangle+\left\langle(0,1),\left(\gamma_{2}, 0\right)\right\rangle+\left\langle(0,0),\left(\gamma_{3}, \exp \left(\gamma_{3}\right)+\delta_{3}\right)\right\rangle \\
& =-1 \\
& =-\frac{1}{2}(1+1+0) \\
& =-\frac{1}{2}\left(\|(0,-1)\|^{2}+\|(0,1)\|^{2}+\|(0,0)\|^{2}\right) \\
& =-\frac{1}{2}\left(\left\|y_{1}\right\|^{2}+\left\|y_{2}\right\|^{2}+\left\|y_{3}\right\|^{2}\right) .
\end{aligned}
$$

and thus (83) holds (again with equality).

Next, using (84) and (85), we obtain

$$
\begin{aligned}
& \mathbf{e}=\left(e_{1}, e_{2}, e_{3}\right)=\left(\left(0, \frac{2}{3}\right),\left(0,-\frac{1}{3}\right),\left(0,-\frac{1}{3}\right)\right) \\
& \mathbf{v}=\left(v_{1}, v_{2}, v_{3}\right)=((0,-1),(0,0),(0,1)) .
\end{aligned}
$$

The vector $\mathbf{v}$ allows us to find the fixed point sets $F_{1}, F_{2}, F_{3}$ (see (3)) via Theorem 9. For instance,

$$
\begin{aligned}
F_{3} & =C_{3} \cap\left(C_{2}+v_{2}\right) \cap\left(C_{1}+v_{1}+v_{2}\right) \\
& =\operatorname{epi}(\exp ) \cap(\mathbb{R} \times\{0\}+(0,0)) \cap(\mathbb{R} \times\{1\}+(0,-1)) \\
& =\operatorname{epi}(\exp ) \cap(\mathbb{R} \times\{0\}) \cap(\mathbb{R} \times\{0\}) \\
& =\varnothing,
\end{aligned}
$$

which again can also be seen geometrically.

\section{Finding the difference vectors for $m \leq 5$ by Banach}

In this section, we discuss an iterative technique to compute $\mathbf{y}$ (given by (38)) which can be used to obtain the difference vector $\mathbf{v}$ via (52). Note that (38) is equivalent to

$$
-\frac{1}{2} \mathbf{y}-\mathbf{T y} \in N_{\overline{\mathbf{C}+\boldsymbol{\Delta}}}^{-1}(\mathbf{y}) .
$$

In this section, let us abbreviate

$$
\mathbf{P}:=P_{\overline{\mathbf{C}+\Delta}}
$$

which is a projector and hence firmly nonexpansive. It follows that Id $-\mathbf{P}$ is also firmly nonexpansive, hence nonexpansive (1-Lipschitz continuous). This allows us to rewrite (115) as $\mathbf{y} \in N_{\overline{\mathbf{C}+\boldsymbol{\Delta}}}\left(-\frac{1}{2} \mathbf{y}-\mathbf{T y}\right) \Leftrightarrow \mathbf{y}+\left(-\frac{1}{2} \mathbf{y}-\right.$ $\mathbf{T y}) \in\left(\mathrm{Id}+N_{\overline{\mathbf{C}+\boldsymbol{\Delta}}}\right)\left(-\frac{1}{2} \mathbf{y}-\mathbf{T y}\right) \Leftrightarrow-\frac{1}{2} \mathbf{y}-\mathbf{T} \mathbf{y}=\mathbf{P}\left(\frac{1}{2} \mathbf{y}-\mathbf{T} \mathbf{y}\right) \Leftrightarrow\left(\frac{1}{2} \mathbf{y}-\mathbf{T y}\right)-\mathbf{y}=\mathbf{P}\left(\frac{1}{2} \mathbf{y}-\mathbf{T y}\right) \Leftrightarrow$

$$
(\operatorname{Id}-\mathbf{P})\left(\frac{1}{2} \mathbf{y}-\mathbf{T y}\right)=\mathbf{y} .
$$

Because we know already that Id $-\mathbf{P}$ is nonexpansive, we can solve (117) by the Banach contraction principle as long as the inner operator

$$
\frac{1}{2} \mathrm{Id}-\mathbf{T}
$$

is a nice Banach contraction, i.e., Lipschitz continuous with a constant strictly less than 1 ! We can determine the operator norm of (118) by analyzing the corresponding matrix in $\mathbb{R}^{m \times m}$. Recall that the singular values are the square roots of the (necessarily nonnegative) eigenvalues of the symmetric matrix associated with $\left(\frac{1}{2} \mathrm{Id}-\mathbf{T}\right)^{*}\left(\frac{1}{2} \mathrm{Id}-\mathbf{T}\right)$. The operator norm is the largest singular value. All this can be found using a symbolic algebra package such as SageMath (or Maple or Mathematica); see Table 1 which provides the squared singular values (with multiplicity) as well as the desired operator norm.

Therefore, when $m \leq 5$, then the fixed point equation (117) can theoretically be solved by the Banach contraction mapping principle. (When $m \geq 7$, the operator norm $\left\|\frac{1}{2} \mathrm{Id}-\mathbf{T}\right\|$ appears to be always strictly larger than 1.) Unfortunately, we do not know of an explicit formula for the projector defined in (116). In practice, one may appeal to Seeger's algorithm [22] for computing $\mathbf{P}$, which we record now: 
Table 1 Computing the operator norm of (118).

\begin{tabular}{lll}
\hline$m$ & eigenvalues of $\left(\frac{1}{2} \operatorname{Id}-\mathbf{T}\right)^{*}\left(\frac{1}{2} \mathrm{Id}-\mathbf{T}\right)$ & $\left\|\frac{1}{2} \mathrm{Id}-\mathbf{T}\right\|$ \\
\hline 2 & $\frac{1}{4}$ (twice) & $\frac{1}{2}=0.5$ \\
3 & $\frac{1}{3}$ (twice), $\frac{1}{4}$ & $\frac{1}{\sqrt{3}} \approx 0.58$ \\
4 & $\frac{1}{2}$ (twice), $\frac{1}{4}$ (twice) & $\frac{1}{\sqrt{2}} \approx 0.71$ \\
5 & $\frac{1}{2}+\frac{1}{2 \sqrt{5}}($ twice $), \frac{1}{2}-\frac{1}{2 \sqrt{5}}$ (twice), $\frac{1}{4}$ & $\sqrt{\frac{1}{2}+\frac{1}{2 \sqrt{5}}} \approx 0.85$ \\
6 & 1 (twice), $\frac{1}{3}$ (twice), $\frac{1}{4}$ (twice) & 1 \\
\hline
\end{tabular}

Fact 12 (Seeger's algorithm). Given

$\mathbf{x} \in \mathbf{X}, \quad$ and $\quad \mathbf{d}_{0} \in \mathbf{X}$

generate sequences $\left(\mathbf{c}_{n}\right)_{n \geq 1}$ and $\left(\mathbf{d}_{n}\right)_{n \geq 1}$ iteratively via

$$
\mathbf{c}_{n}:=P_{\mathbf{C}}\left(\mathbf{x}-\mathbf{d}_{n-1}\right), \quad \mathbf{d}_{n}:=P_{\boldsymbol{\Delta}}\left(\mathbf{x}-\mathbf{c}_{n}\right) .
$$

Then

$$
\mathbf{c}_{n}+\mathbf{d}_{n} \rightarrow P \overline{\mathbf{C}+\boldsymbol{\Delta}}(\mathbf{x})=\mathbf{P}(\mathbf{x})
$$

\section{Finding the difference vectors by forward-backward}

In this section, we sketch another approach to numerically compute the difference vectors. We begin by revisiting (38) as a primal problem:

- Proposition 13. We interpret

$$
0 \in N_{\overline{\mathbf{C}+\boldsymbol{\Delta}}}^{-1}(\mathbf{y})+\left(\frac{1}{2} \operatorname{Id}+\mathbf{T}\right)(\mathbf{y}),
$$

which is (38) and for which the solution $\mathbf{y}$ is unique, as an Attouch-Théra primal problem for the pair $\left(N_{\overline{\mathbf{C}+\boldsymbol{\Delta}}}^{-1}, \frac{1}{2} \mathrm{Id}+\mathbf{T}\right)$. Then $\frac{1}{2} \mathrm{Id}+\mathbf{T}$ is $\frac{1}{2}$-strongly monotone and $\left(\frac{1}{2} \mathrm{Id}+\mathbf{T}\right)^{-1}$ is $\frac{1}{2}$-cocoercive. Moreover, the AttouchThéra dual problem of (120) is

$$
0 \in N \overline{\mathbf{C}+\boldsymbol{\Delta}}(\mathbf{x})+\left(\frac{1}{2} \operatorname{Id}+\mathbf{T}\right)^{-1}(\mathbf{x})
$$

and the solution set of (121) is the singleton

$$
\{\mathbf{e}\}=\boldsymbol{\Delta}^{\perp} \cap \operatorname{Fix}\left(P_{\overline{\mathbf{C}+\boldsymbol{\Delta}}} \mathbf{R}\right) .
$$

Proof. Because $\mathbf{T}$ is skew (see (23)), it follows that $\frac{1}{2} \mathrm{Id}+\mathbf{T}$ is $\frac{1}{2}$-strongly monotone. By [10, Example 22.7], $\left(\frac{1}{2} \mathrm{Id}+\mathbf{T}\right)^{-1}$ is $\frac{1}{2}$-cocoercive. Because $\mathbf{T}$ is linear, the Attouch-Théra dual of (120) with respect to the pair $\left(N_{\overline{\mathbf{C}+\boldsymbol{\Delta}}}^{-1}, \frac{1}{2} \mathrm{Id}+\mathbf{T}\right)$ is indeed (121). We can pass from $\mathbf{y}$, the unique solution of (120), to the set of solutions of (121) via $N_{\overline{\mathbf{C}+\boldsymbol{\Delta}}}^{-1}(\mathbf{y}) \cap-\left(\frac{1}{2} \operatorname{Id}+\mathbf{T}\right)(\mathbf{y})$ (see [9, Proposition 2.4]). Because $\mathbf{T}$ is single-valued, this implies that the unique solution to $(121)$ is

$$
\mathbf{x}=N_{\overline{\mathbf{C}+\boldsymbol{\Delta}}}^{-1}(\mathbf{y}) \cap-\left(\frac{1}{2} \mathrm{Id}+\mathbf{T}\right)(\mathbf{y})=-\frac{1}{2} \mathbf{y}-\mathbf{T y}=\mathbf{e},
$$

where we used (40) for the last equality. Now consider (121) again. We rewrite this, using (123), (32) and (40) as

$$
0 \in N_{\overline{\mathbf{C}+\boldsymbol{\Delta}}}(\mathbf{e})+\left(\operatorname{Id}-\mathbf{R}+2 P_{\Delta}\right)(\mathbf{e})=N_{\overline{\mathbf{C}+\boldsymbol{\Delta}}}(\mathbf{e})+(\operatorname{Id}-\mathbf{R})(\mathbf{e}),
$$

or as $\mathbf{e}=P_{\overline{\mathbf{C}+\boldsymbol{\Delta}}}(\mathbf{R e}) \in \overline{\mathbf{C}+\boldsymbol{\Delta}}$ which yields (122).

Theorem 14. Let $\gamma \in] 0,1\left[\right.$, let $\mathbf{x}_{0} \in \mathbf{X}$, and generate a sequence $\left(\mathbf{x}_{n}\right)_{n \in \mathbb{N}}$ via

$$
\begin{aligned}
\mathbf{x}_{n+1} & =P_{\overline{\mathbf{C}+\boldsymbol{\Delta}}}\left(\mathbf{x}_{n}-\gamma\left(\frac{1}{2} \operatorname{Id}+\mathbf{T}\right)^{-1} \mathbf{x}_{n}\right) \\
& =P_{\overline{\mathbf{C}+\boldsymbol{\Delta}}}\left((1-\gamma) \mathbf{x}_{n}+\gamma \mathbf{R} \mathbf{x}_{n}-2 \gamma P_{\boldsymbol{\Delta}} \mathbf{x}_{n}\right) .
\end{aligned}
$$


Then

$$
\begin{aligned}
& \mathbf{x}_{n} \rightarrow \mathbf{e} \\
& \mathbf{R} \mathbf{x}_{n}-\mathbf{x}_{n}-2 P_{\Delta} \mathbf{x}_{n} \rightarrow \mathbf{y} \\
& \mathbf{R}^{*} \mathbf{x}_{n}-\mathbf{x}_{n} \rightarrow \mathbf{v}
\end{aligned}
$$

Proof. Set $\mathbf{A}:=N_{\overline{\mathbf{C}+\boldsymbol{\Delta}}}$. Also set $\mathbf{B}:=\left(\frac{1}{2} \mathrm{Id}+\mathbf{T}\right)^{-1}$, which is $\beta$-cocoercive, with $\beta=\frac{1}{2}$, by Proposition 13 . Then $\gamma \in] 0,2 \beta\left[\right.$. Now set $\delta:=2-\gamma /(2 \beta)=2-\gamma>1$ and $\lambda:=\lambda_{n} \equiv 1$. Then $\lambda_{n}\left(\delta-\lambda_{n}\right) \equiv \delta-1>0$ and thus $\sum_{n \in \mathbb{N}} \lambda_{n}\left(\delta-\lambda_{n}\right)=+\infty$. We now apply [10, Theorem 26.14] on the forward-backward algorithm applied to the problem (121). Note that (125a) is precisely the forward-backward algorithm with the parameters just defined because of [10, Remark 26.15] and $\mathbf{x}_{n+1}=J_{\gamma \mathbf{A}}\left(\mathbf{x}_{n}-\gamma \mathbf{B} \mathbf{x}_{n}\right)$. The alternative formula (125b) follows from (32). Using [10, Theorem 26.14(i)\&(ii)] and Proposition 13, we have $\mathbf{x}_{n} \rightarrow \mathbf{e}$ and

$$
\mathbf{B} \mathbf{x}_{n}=\left(\frac{1}{2} \mathrm{Id}+\mathbf{T}\right)^{-1} \mathbf{x}_{n} \rightarrow\left(\frac{1}{2} \mathrm{Id}+\mathbf{T}\right)^{-1} \mathbf{e}=-\mathbf{y} .
$$

(The fact that $\mathbf{B} \mathbf{x}_{n} \rightarrow-\mathbf{y}$ and not $\mathbf{y}$ stems from the fact that the dual problem in [10, Chapter 26] differs from the one in this paper by a negative sign.) Now (129) and (32) yield (127). Next, (129) and the fact that $\mathbf{T}$ is continuous and single-valued yields

$$
\mathbf{x}_{n}=\left(\frac{1}{2} \operatorname{Id}+\mathbf{T}\right)\left(\frac{1}{2} \operatorname{Id}+\mathbf{T}\right)^{-1} \mathbf{x}_{n} \rightarrow\left(\frac{1}{2} \operatorname{Id}+\mathbf{T}\right)\left(\frac{1}{2} \operatorname{Id}+\mathbf{T}\right)^{-1} \mathbf{e}=\mathbf{e}
$$

and so (126) is verified. To check (128), apply the continuous operator $\mathbf{R}^{*}-$ Id to (130) and recall (49).

Remark 15. Theorem 14 is a powerful result for computing $\mathbf{e}, \mathbf{y}, \mathbf{v}$ as strong limits of sequence. As in Section 6 , the numerical difficulty lies in the computation of $P_{\overline{\mathbf{C}+\boldsymbol{\Delta}}}$; however, Seeger's algorithm (see Fact 12) may be used to approximate this projection.

- Remark 16. Theorem 14 allows for flexibility of the parameter $\gamma \in] 0,1[$. Perhaps the most natural choice is

$$
\gamma=\frac{1}{2}
$$

however, let us point out an intriguing other choices, namely

$$
\gamma=\frac{m}{m+2}
$$

With the latter choice and (21), the inner (forward) operator in (125b) turns into

$$
\begin{aligned}
(1-\gamma) \operatorname{Id}+\gamma \mathbf{R}-2 \gamma P_{\boldsymbol{\Delta}} & =\frac{2}{m+2} \mathrm{Id}+\frac{m}{m+2} \mathbf{R}-\frac{2 m}{m+2} P_{\boldsymbol{\Delta}} \\
& =\frac{2}{m+2} \mathrm{Id}+\frac{m}{m+2} \mathbf{R}-\frac{2 m}{m+2} \frac{1}{m} \sum_{k=0}^{m-1} \mathbf{R}^{k} \\
& =\frac{m-2}{m+2} \mathbf{R}-\frac{2}{m+2} \sum_{k=2}^{m-1} \mathbf{R}^{k},
\end{aligned}
$$

which is Lipschitz continuous with constant $3(m-2) /(m+2)$ because $\mathbf{R}$ is an isometry. We point out the cases when $m=2$ and $m=3$, for which $\gamma=1 / 2$ and $\gamma=3 / 5$ respectively, and (133) turns into

$$
\left[m=2 \text { and } \gamma=\frac{1}{2}\right] \Rightarrow(1-\gamma) \operatorname{Id}+\gamma \mathbf{R}-2 \gamma P_{\Delta} \equiv 0 \text { is } 0 \text {-Lipschitz }
$$

and

$$
\left[m=3 \text { and } \gamma=\frac{3}{5}\right] \Rightarrow(1-\gamma) \operatorname{Id}+\gamma \mathbf{R}-2 \gamma P_{\boldsymbol{\Delta}}=\frac{1}{5} \mathbf{R}-\frac{2}{5} \mathbf{R}^{2} \text { is } \frac{3}{5} \text {-Lipschitz. }
$$

Note that (134) looks at first puzzling because then (125b) turns into $\mathbf{x}_{n+1}=P_{\overline{\mathbf{C}+\boldsymbol{\Delta}}}(0)$ and so (126) yields $\mathbf{e}=P_{\overline{\mathbf{C}+\boldsymbol{\Delta}}}(0)$; however, we already observed this directly in (61). 


\section{Conclusion and future work}

Using the framework of monotone operator theory, we resolved the geometry conjecture completely. We obtained alternative descriptions of the set of cycles $\mathbf{Z}$. We also sketched numerical approaches for the computation of the difference vector $\mathbf{v}$ by using Seeger's algorithm.

Turning to future research, it is desirable to devise algorithms for computing $\mathbf{v}$ without having to employ Seeger's algorithm. Moreover, it is interesting to extend the results in this paper from projectors to (underrelaxed) projectors or even proximal mappings. We have taken steps in this direction, and initial progress appears to be quite promising [2].

\section{References}

1 Salihah Alwadani, Heinz H. Bauschke, Julian P. Revalski, and Xianfu Wang. Resolvents and Yosida approximations of displacement mappings of isometries. Set-Valued Var. Anal., 2021. https://link.springer.com/article/10. $1007 / \mathrm{s} 11228-021-00584-2$.

2 Salihah Alwadani, Heinz H. Bauschke, and Xianfu Wang. Attouch-Théra duality, generalized cycles and gap vectors. https://arxiv.org/abs/2101.05857, to appear in SIAM J. Optim., 2021.

3 Hedy Attouch and Michel Théra. A general duality principle for the sum of two operators. J. Convex Anal., 3(1):1-24, 1996.

4 Jean-Bernard Baillon, Patrick L. Combettes, and Roberto Cominetti. There is no variational characterization of the cycles in the method of periodic projections. J. Funct. Anal., 262(1):400-408, 2012.

5 Jean-Bernard Baillon, Patrick L. Combettes, and Roberto Cominetti. Asymptotic behavior of compositions of underrelaxed nonexpansive operators. J. Dyn. Games, 1(3):331-346, 2014.

6 Heinz H. Bauschke and Jonathan M. Borwein. On the convergence of von Neumann's alternating projection algorithm for two sets. Set-Valued Anal., 1:185-212, 1993.

7 Heinz H. Bauschke and Jonathan M. Borwein. Dykstra's alternating projection algorithm for two sets. J. Approx. Theory, 79:418-443, 1994.

8 Heinz H. Bauschke, Jonathan M. Borwein, and Adrian S. Lewis. The method of cyclic projections for closed convex sets in Hilbert space. In Recent developments in optimization theory and nonlinear analysis, volume 104 of Contemporary Mathematics, pages 1-38. American Mathematical Society, 1997.

9 Heinz H. Bauschke, Radu I. Bot, Warren L. Hare, and Walaa M. Moursi. Attouch-Théra duality revisited: paramonotonicity and operator splitting. J. Approx. Theory, 164(8):1065-1084, 2012.

10 Heinz H. Bauschke and Patrick L. Combettes. Convex analysis and monotone operator theory in Hilbert spaces. CMS Books in Mathematics/Ouvrages de Mathématiques de la SMC. Springer, second edition, 2017.

11 Heinz H. Bauschke, Victoria Martín-Márquez, Sarah M. Moffat, and Xianfu Wang. Compositions and convex combinations of asymptotically regular firmly nonexpansive mappings are also asymptotically regular. Fixed Point Theory Appl., 2012, 2012.

12 Haïm Brézis. Operateurs maximaux monotones et semi-groupes de contractions dans les espaces de Hilbert, volume 5 of North-Holland Mathematics Studies. North-Holland, 1973.

13 Regina S. Burachik and Alfredo N. Iusem. Set-valued mappings and enlargement of monotone operators, volume 8 of Springer Optimization and Its Applications. Springer, 2008.

14 Charles L. Byrne. Signal processing. A mathematical approach. A K Peters, 2005.

15 Charles L. Byrne. Applied iterative methods. A K Peters, 2008.

16 Yair Censor and Maroun Zaknoon. Algorithms and convergence results of projection methods for inconsistent feasibility problems: A review. Pure Appl. Funct. Anal., 3(4):565-586, 2018.

17 Ward Cheney and Allen A. Goldstein. Proximity maps for convex sets. Proc. Am. Math. Soc., 10:448-450, 1959.

18 Patrick L. Combettes and Jean-Christophe Pesquet. Fixed point strategies in data science. IEEE Trans. Signal Process., 2021. https://doi.org/10.1109/TSP.2021.3069677.

19 Roberto Cominetti, Vera Roshchina, and Andrew Williamson. A counterexample to De Pierro's conjecture on the convergence of under-relaxed cyclic projections. Optimization, 68(1):3-12, 2019.

20 Alvaro R. De Pierro. From parallel to sequential projection methods and vice versa in convex feasibility: Results and conjectures. In Inherently Parallel Algorithms in Feasibility and Optimization and their Applications, volume 8 of Studies in Computational Mathematics, pages 369-379. North-Holland, 2001.

21 Alfredo N. Iusem. On some properties of paramonotone operators. J. Convex Anal., 5(2):269-278, 1998.

22 Alberto Seeger. Alternating projection and decomposition with respect to two convex sets. Math. Jap., 47(2):273-280, 1998.

23 Stephen Simons. Minimax and monotonicity, volume 1693 of Lecture Notes in Mathematics. Springer, 1998.

24 Stephen Simons. From Hahn-Banach to monotonicity, volume 1693 of Lecture Notes in Mathematics. Springer, 2nd expanded edition, 2008. 